\title{
A human mutated gene is guillotining spermatozoa
}

\author{
Jesús del Mazo ${ }^{1}$, Miguel A. Brieño-Enríquez ${ }^{2}$, Eduardo Larriba ${ }^{1}$ \\ ${ }^{1}$ Department of Cellular and Molecular Biology, Centro de Inestigaciones Biológicas (CSIC), Madrid, Spain; ${ }^{2}$ Department of Biomedical Sciences, \\ Center for Reproductive Genomics, Cornell University, Ithaca, NY, USA \\ Correspondence to: Jesús del Mazo. Department of Cellular and Molecular Biology, Centro de Investigaciones Biológicas (CSIC), Ramiro de Maeztu 9, \\ Madrid 28040, Spain. Email: jdelmazo@cib.csic.es. \\ Comment on: Li L, Sha Y, Wang X, et al. Whole-exome sequencing identified a homozygous BRDT mutation in a patient with acephalic spermatozoa. \\ Oncotarget 2017;8:19914-22.
}

Submitted Feb 19, 2018. Accepted for publication Mar 02, 2018.

doi: 10.21037/tcr.2018.03.06

View this article at: http://dx.doi.org/10.21037/tcr.2018.03.06

At reproductive age, about $13 \%$ of the general population suffer from fertility disturbances, with at least a third of them attributable to male factors (1). Recent meta-analysis of male fertility indicated a decline accumulative of over $1 \%$ per year in semen quality (2), most likely due to environmental factors, such as, exposure to endocrine disrupting compounds (3). In addition to this alarming trend in human fertility caused by environmental conditions, multiple cases of male infertility with phenotypes of spermatozoa alterations in otherwise azoospermic men, are from genetic origin, including deletions and mutations, mainly with loci in the $\mathrm{Y}$ chromosome (4). Spermatogenesis is of a polygenic nature and the morphogenetic processes to generate functional spermatozoa require complex regulations. During spermatogenic differentiation, the proper development of the gonads during early embryogenesis is necessary to produce healthy spermatozoa during adulthood. Abnormal regulation of embryogenesis by genetic factors such as single nucleotide polymorphisms or mutation can induce male infertility by causing spermatozoa deformities and germ cell tumors (5).

Disruption of spermatogenesis is detected in some familial clustering causing infertility or subfertility that strongly suggested genetic contribution and mutation transmission as the cause of such male fertility dysfunction $(6,7)$. However, few single gene mutations have been identified in humans causing male infertility, mostly associated with asthenozoospermia (8). Although, some knock-out mammalian animal models of male infertility have been generated in attempt to mimic some familial multiple anomalies that produced developmentally impaired sperm (9), most of these cases are limited to single mutations detected in men. However, mutant mice do not fully display comparable phenotypes. Given this limitation in male mice, it makes it difficult to study the thousands of genes that are estimated to be involved in spermatogenesis and functional spermatozoa differentiation.

Both reverse and forward genetic approaches have been established in the mouse patterns of sperm differentiation (10). However, to establish gene mutations in humans associated with male infertility, population analysis in affected families should continue to be performed. Recent molecular and bioinformatics tools used for genetic analysis will allow for a wider and efficient search of mutations associated with specific phenotypes causing fertility disturbances due to spermatozoa anomalies. Generation of knockout (KO) mice by point mutation utilizing CRISPR/Cas9 system as a genome editing tool to target mutations that are related to male infertility, has allowed for further understanding the effects of such mutations and understanding the involved molecular pathways (11).

Technological advances in DNA sequencing are accelerating exponentially our knowledge of human genetics and genomics. Next-generation sequencing (NGS) technology, also known as high-throughput sequencing, allows for sequencing DNA much more quickly, is more cost effect and with higher-accuracy. An example of NGS is RNA-seq, a substitute for DNA microarray, which can now present a more accurate quantification of gene expression and mRNA isoform levels (12). Another advantage of NGS technology is the versatility offered by the platforms and sequencing protocols. Using these tools has enhanced the capacity to sequencing all gene coding regions present in the human genome, obtaining the whole exon regions of all 
protein coding genes, and extending the targets to functional non-coding elements such as 3'UTR or miRNA (13). Identify genetic variants that alter protein sequences is the goal of whole-exome sequencing (WES). WES is extensively used in the identification of novel mutations for known disease phenotypes. WES has some advantages compared to whole genome sequencing (WGS). One advantage is that the coding region in the human genome does not exceed $2 \%$ of all its base length, therefore bases to be sequenced in a WES is much lower than sequencing necessary for WGS. This allows a reduction in sequencing costs per sample and decreases the processing time. In addition, the amount of data generated by WES is smaller than by WGS, so handling analysis and storage requires less computational resources and bioinformatics infrastructure.

Recently Li et al. identified a homozygous mutation in an infertile male by WES in a consanguineous family (14). The mutation was found in the bromodomain testis associated gene (BRDT), which has genetic origin as demonstrated by the familial study. The phenotypic consequence of the missense mutation was associated to a very rare cause of male infertility, acephalic or "decapitated" spermatozoa. Using a RNA-seq in an experimental approach on male teratocarcinoma cell line comparing wild type expression and transfected cells with the specific mutation, the authors showed that about 900 genes were detected as differentially expressed. Gene Ontology (GO) analysis of the deregulated genes detected multiple biological processes that could be involved in the pathology of acephalic spermatozoa detected in the homozygous patient. However, due to the wide number and the generic biological functions of deregulated genes it is not possible to pinpoint the precise weight of each deregulated gene that is a direct consequence of a mutation in $B R D T$ with the consequence of this rare sperm pathology. BRDT is a gene widely studied, the protein contains two bromodomain motifs and a cluster of proline, glutamic acid, serine, and threonine residues, characteristic of proteins that undergo rapid intracellular degradation (Ensembl: ENSG00000137948). KO mice bearing a loss of the BRDT gene showed severe alteration of spermatogenesis, even at the level of meiotic arrest (15). In the detected mutation in the patient, $\mathrm{Li}$ et al. suggested a gain-of-function, which is putatively in conflict with the expected high turnover of this protein. Other SNPs that theoretically did not affect the activity of the protein are reported in azoospermic men (16). Taken together these data indicate that a precise equilibrium in the level of $B R D T$ is necessary for correct spermatogenesis, including during the last stages of spermiogenesis. In the detected mutation the acephalic spermatozoa were detected as a single sperm tail, which suggested that the last spermiogenesis stages, including the initial formation of the axonema and formation of sperm tail took place. This indicated that trimming of the head could generate the acephalic spermatozoa. In addition to the acephalic spermatozoa, there was also a loss of mitochondria that was observed. However, the origin of the loss of the mitochondria is unknown. The authors indicated the potential role of the glycine 928 of the P-TEFb binding domain of the BRDT protein. However, in the absence of histological testis data a more accurate dissection of the role of this specific amino acid was not possible. New experimental approaches should be designed to assess the role of BRDT. In this respect, for example specific mutations in mouse models can be generated by genome editing using the CRISPR/Cas9 system. Such models would lead to further understanding whether the mutation detected in the Li et al. study is associated with alterations in the interaction between Golgi complex and centrioles during spermatogenesis, as the authors propose. However, another potential explanation raises in and abnormal centrosome function that is clearly related to different types of teratozoospermia (17). Curiously, the expression of BRDT was also detected as a potential marker in a relative high proportion of non-small cell lung cancer cases (18). Consequently, the generation of such mouse models of specific BRDT mutations may also deepen additional pathologies including lung cancer.

\section{Acknowledgments}

We want to thank Catalina Pereira for proofreading the manuscript.

Funding: The work in the $\mathrm{J}$ del Mazo laboratory was supported by the grants from the Ministerio de Industria Economía y Competitividad, Spain (BFU2013-42164-R and BFU2017-87095-R).

\section{Footnote}

Provenance and Peer Review: This article was commissioned and reviewed by the Section Editor Weijun Jiang (Nanjing Normal University, Department of Reproductive and Genetics, Institute of Laboratory Medicine, Jinling Hospital, Nanjing University School of Medicine, Nanjing, China). 
Conflicts of Interest: All authors have completed the ICMJE uniform disclosure form (available at http://dx.doi. org/10.21037/tcr.2018.03.06). The authors have no conflicts of interest to declare.

Ethical Statement: The authors are accountable for all aspects of the work in ensuring that questions related to the accuracy or integrity of any part of the work are appropriately investigated and resolved.

Open Access Statement: This is an Open Access article distributed in accordance with the Creative Commons Attribution-NonCommercial-NoDerivs 4.0 International License (CC BY-NC-ND 4.0), which permits the noncommercial replication and distribution of the article with the strict proviso that no changes or edits are made and the original work is properly cited (including links to both the formal publication through the relevant DOI and the license). See: https://creativecommons.org/licenses/by-nc-nd/4.0/.

\section{References}

1. Kumar N, Singh AK. Trends of male factor infertility, an important cause of infertility: A review of literature. J Hum Reprod Sci 2015;8:191-6.

2. Levine H, Jørgensen N, Martino-Andrade A, et al. Temporal trends in sperm count: a systematic review and meta-regression analysis. Hum Reprod Update 2017;23:646-59.

3. del Mazo J, Brieño-Enríquez MA, García-López J, et al. Endocrine disruptors, gene deregulation and male germ cell tumors. Int J Dev Biol 2013;57:225-39.

4. Silber SJ. The $\mathrm{Y}$ chromosome in the era of intracytoplasmic sperm injection: a personal review. Fertil Steril 2011;95:2439-48.e1-5.

5. van der Zwan YG, Rijlaarsdam MA, Rossello FJ, et al. Seminoma and embryonal carcinoma footprints identified by analysis of integrated genome-wide epigenetic and expression profiles of germ cell cancer cell lines. PLoS One 2014;9:e98330.

6. Gianotten J, Westerveld GH, Leschot NJ, et al. Familial clustering of impaired spermatogenesis: no evidence for a common genetic inheritance pattern. Hum Reprod
2004;19:71-6.

7. Tuerlings JH, van Golde RJ, Oudakker AR, et al. Familial oligoasthenoteratozoospermia: evidence of autosomal dominant inheritance with sex-limited expression. Fertil Steril 2002;77:415-8.

8. O'Flynn O'Brien KL, Varghese AC, Agarwal A. The genetic causes of male factor infertility: a review. Fertil Steril 2010;93:1-12.

9. Juneja SC, van Deursen JM. A mouse model of familial oligoasthenoteratozoospermia. Hum Reprod 2005;20:881-93.

10. Jamsai $\mathrm{D}, \mathrm{O}$ 'Bryan MK. Mouse models in male fertility research. Asian J Androl 2011;13:139-51.

11. Abbasi F, Miyata H, Ikawa M. Revolutionizing male fertility factor research in mice by using the genome editing tool CRISPR/Cas9. Reprod Med Biol 2017;17:3-10.

12. Wang Z, Gerstein $M$, Snyder M. RNA-Seq: a revolutionary tool for transcriptomics. Nat Rev Genet 2009;10:57-63.

13. Warr A, Robert C, Hume D, et al. Exome Sequencing: Current and Future Perspectives. G3 (Bethesda) 2015;5:1543-50.

14. Li L, Sha Y, Wang X, et al. Whole-exome sequencing identified a homozygous BRDT mutation in a patient with acephalic spermatozoa. Oncotarget 2017;8:19914-22.

15. Gaucher J, Boussouar F, Montellier E, et al. Bromodomain-dependent stage-specific male genome programming by Brdt. EMBO J 2012;31:3809-20.

16. Barda S, Yogev L, Paz G, et al. BRDT gene sequence in human testicular pathologies and the implication of its single nucleotide polymorphism (rs3088232) on fertility. Andrology 2014;2:641-7.

17. Terada Y, Schatten G, Hasegawa H, et al. Essential roles of the sperm centrosome in human fertilization: developing the therapy for fertilization failure due to sperm centrosomal dysfunction. Tohoku J Exp Med 2010;220:247-58.

18. Scanlan MJ, Altorki NK, Gure AO, et al. Expression of cancer-testis antigens in lung cancer: definition of bromodomain testis-specific gene (BRDT) as a new CT gene, CT9. Cancer Lett 2000;150:155-64.
Cite this article as: del Mazo J, Brieño-Enríquez MA, Larriba E. A human mutated gene is guillotining spermatozoa. Transl Cancer Res 2018;7(Suppl 4):S466-S468. doi: 10.21037/ tcr.2018.03.06 